\section{Complicated asymptotic behavior of solutions for a porous medium equation with nonlinear sources}

Liangwei Wang $^{1 *}$ and Jingxue Yin ${ }^{2}$

${ }^{*}$ Correspondence:
wanglw08@163.com
1School of Mathematics and
Statistics, Chongqing Three Gorges
University, Wanzhou, China
Full list of author information is
available at the end of the article

${ }^{*}$ Correspondence: wanglw08@163.com University, Wanzhou, China available at the end of the article

\begin{abstract}
In this paper, we investigate the complicated asymptotic behavior of the solutions to the Cauchy problem of a porous medium equation with nonlinear sources when the initial value belongs to a weighted $L^{\infty}$ space.
\end{abstract}

AMS Subject Classification: 35K55; 35B40

Keywords: complexity; asymptotic behavior; porous medium equation

\section{Introduction}

In this paper, we consider the asymptotic behavior of solutions for the Cauchy problem of the porous medium equation with nonlinear sources

$$
\begin{aligned}
& \frac{\partial u}{\partial t}-\Delta u^{m}=u^{p}, \quad \text { in } \mathbb{R}^{N} \times(0, \infty), \\
& u(x, 0)=u_{0}(x), \quad \text { in } \mathbb{R}^{N},
\end{aligned}
$$

where $m, p>1$ and $u_{0} \in L^{\infty}\left(\rho_{\sigma}\right) \equiv\left\{\varphi ; \varphi \rho_{\sigma} \in L^{\infty}\left(\mathbb{R}^{N}\right)\right\}$ with $\rho_{\sigma}(x)=\left(1+|x|^{2}\right)^{\frac{\sigma}{2}}$.

It is well known that any positive solutions of problem (1.1)-(1.2) blow up in finite time if $1<p \leq p_{c} \equiv m+\frac{2}{N}$ [1-3], while positive global solutions do exist if $p>p_{c}$ [4-7]. In 2000, Mukai, Mochizuki and Huang in [6] found that if $p>m+\frac{2}{N}$ and $\frac{2}{p-m}<\sigma<N$ and $0 \leq \varphi \in C_{b}\left(\mathbb{R}^{N}\right)$ satisfies $\lim \sup _{|x| \rightarrow \infty}|x|^{\sigma} \varphi(x)<\infty$, then there exists a constant $\eta(\varphi)>0$ such that for $0<\eta<\eta(\varphi)$, the solutions $u(x, t)$ of problem (1.1)-(1.2) with the initial value $u_{0}=\eta \varphi$ are global and the following estimate holds:

$$
\|u(t)\|_{L^{\infty}\left(\mathbb{R}^{N}\right)} \leq C t^{-\frac{\sigma}{\sigma(m-1)+2}} .
$$

Moreover, if $\lim _{|x| \rightarrow \infty}|x|^{\sigma} \varphi(x)=M_{0}>0$, then

$$
t^{\frac{\sigma}{\sigma(m-1)+2}} u\left(t^{\frac{1}{\sigma(m-1)+2}} x, t\right) \stackrel{t \rightarrow \infty}{\longrightarrow} S(1) w_{0}(x)
$$

(0) 2013 Wang and Yin; licensee Springer. This is an Open Access article distributed under the terms of the Creative Commons Attribution License (http://creativecommons.org/licenses/by/2.0), which permits unrestricted use, distribution, and reproduction in any medium, provided the original work is properly cited. 
uniformly on $\mathbb{R}^{N}$, where $w_{0}(x)=M|x|^{-\sigma}$. Here $S(t)$ is a semigroup generated by the Cauchy problem of the porous medium equation

$$
\begin{aligned}
& \frac{\partial w}{\partial t}-\Delta w^{m}=0, \quad \text { in } \mathbb{R}^{N} \times(0, \infty), \\
& w(x, 0)=w_{0}(x), \quad \text { in } \mathbb{R}^{N}
\end{aligned}
$$

and $w_{0}(x)=\eta M_{0}|x|^{-\sigma}$.

On the other hand, regarding problem (1.4)-(1.5), in 2002, Vázquez and Zuazua [8] found that for any bounded sequence $\left\{\varphi_{n}\right\}_{n=1}^{\infty}$ in $L^{\infty}\left(\mathbb{R}^{N}\right)$, there exists an initial value $u_{0} \in$ $L^{\infty}\left(\mathbb{R}^{N}\right)$ and a sequence $t_{n_{k}} \rightarrow \infty$ as $k \rightarrow \infty$ such that $\lim _{k \rightarrow \infty} S\left(t_{n_{k}}\right) u_{0}\left(t_{n_{k}}^{\frac{1}{2}} x\right)=S(1) \varphi_{n}(x)$ uniformly on any compact subsets of $\mathbb{R}^{N}$. In our previous papers [9], for any bounded sequence $\left\{\varphi_{n}\right\}_{n=1}^{\infty}$ in $C_{0}^{+}\left(\mathbb{R}^{N}\right) \equiv\left\{\varphi \in C_{0}\left(\mathbb{R}^{N}\right) ; \phi(x) \geq 0\right\}$, we have shown that there exists an initial value $u_{0} \in C_{0}\left(\mathbb{R}^{N}\right)$ and a sequence $t_{n_{k}} \rightarrow \infty$ as $k \rightarrow \infty$ such that

$$
\lim _{k \rightarrow \infty} t_{n_{k}}^{\frac{\mu}{2}} S\left(t_{n_{k}}\right) u_{0}\left(t_{n_{k}}^{\beta} x\right)=S(1) \varphi_{n}(x)
$$

uniformly on $\mathbb{R}^{N}$, where $0<\mu<\frac{N}{N(m-1)+2}$ and $\beta=\frac{2-\mu(m-1)}{4}$. For more details on the study of complicated asymptotic behavior of solutions for the heat equation and other evolution equations, we refer the readers to [10-14].

In this paper, we are quite interested in the above mentioned same topic for the equation with strongly nonlinear sources, namely equation (1.1) with $p>m+\frac{2}{N}$. We will show that for any $M>0$, there is a constant $\eta(M)$ and an initial value $u_{0} \in C_{\eta(M)}^{\sigma,+} \equiv\left\{\varphi \in C\left(\mathbb{R}^{N}\right) ; \varphi \in\right.$ $\left.B_{\eta(M)}^{\sigma,+}\right\}$ with $\frac{2}{p-m}<\sigma<N$ such that for any $\varphi \in C_{\eta(M)}^{\sigma,+}$, there exists a sequence $t_{n} \rightarrow \infty$ as $n \rightarrow \infty$ satisfying

$$
\lim _{n \rightarrow \infty} t_{n}^{\frac{\sigma}{\sigma(m-1)+2}} S\left(t_{n}\right) u_{0}\left(t_{n}^{\frac{1}{\sigma(m-1)+2}} x\right)=S(1) \varphi(x)
$$

uniformly on $\mathbb{R}^{N}$. Here $B_{\eta(M)}^{\sigma,+} \equiv\left\{\phi=\eta \varphi ; 0 \leq \varphi \in L^{\infty}\left(\rho_{\sigma}\right),\left\|\left(1+|\cdot|^{2}\right)^{\frac{\sigma}{2}} \varphi(\cdot)\right\|_{L^{\infty}\left(\mathbb{R}^{N}\right)} \leq\right.$ $M$ and $0 \leq \eta \leq \eta(M)\}$. For this purpose, we first show that if the initial value $u_{0} \in B_{\eta(M)}^{\sigma,+}$, then the solutions $u(x, t)$ are global and satisfy

$$
u(x, t) \leq C\left(1+t^{\frac{2}{\sigma(m-1)+2}}+|x|^{2}\right)^{-\frac{\sigma}{2}} .
$$

One can easily see that (1.6) captures (1.3). From this, we can follow the framework by Kamin and Peletier [15] to prove that

$$
\lim _{t \rightarrow \infty} t^{\frac{1}{\sigma(m-1)+2}}\left\|u\left(t^{\frac{\sigma}{\sigma(m-1)+2}} \cdot, t\right)-S(t) u_{0}\left(t^{\frac{1}{\sigma(m-1)+2}} \cdot\right)\right\|_{L^{\infty}\left(\mathbb{R}^{N)}\right.}=0
$$

So, we can get our results by following the framework in [9] and using (1.6)-(1.7).

The rest of this paper is organized as follows. The next section is devoted to giving a sufficient condition for the global existence of solutions for problem (1.1)-(1.2) and the upper bounded estimates on these solutions. In the last section, we investigate the complicated asymptotic behavior of solutions. 


\section{Preliminaries and estimates}

In this section we state the definition of a weak solution of problem (1.1)-(1.2) and give the upper bounded estimates on the global solutions. We begin with the definition of the weak solution of problem (1.1)-(1.2).

Definition 2.1 $[16,17]$ By a weak solution of problem (1.1)-(1.2) in $\mathbb{R}^{N} \times[0, T)$, we mean a function $u(x, t)$ in $\mathbb{R}^{N} \times[0, T)$ such that

1. $u(x, t) \geq 0$ in $\mathbb{R}^{N} \times[0, T)$ and $u(x, t) \in C\left(\mathbb{R}^{N} \times(0, \tau]\right)$ for each $0<\tau<T$.

2. For $0<\tau<T$ and any nonnegative $\varphi(x, t) \in C^{2,1}\left(\mathbb{R}^{N} \times[0, T)\right)$ which vanishes for large $|x|$, the following equation holds:

$$
\begin{aligned}
\int_{\mathbb{R}^{N}} u(x, \tau) \varphi(x, \tau) \mathrm{d} x-\int_{\mathbb{R}^{N}} u_{0}(x) \varphi(x, 0) \mathrm{d} x \\
=\int_{0}^{\tau} \int_{\mathbb{R}^{N}} u^{m}(x, t) \Delta \varphi(x, t) \mathrm{d} x \mathrm{~d} t \\
\quad+\int_{0}^{\tau} \int_{\mathbb{R}^{N}} u(x, t) \varphi_{t}(x, t) \mathrm{d} x \mathrm{~d} t+\int_{0}^{\tau} \int_{\mathbb{R}^{N}} u^{p}(x, t) \varphi(x, t) \mathrm{d} x \mathrm{~d} t .
\end{aligned}
$$

A supersolution [or subsolution] is similarly defined with equality of (2.1) replaced by $\geq$ [or $\leq$ ]. The weak solutions for problem (1.4)-(1.5) can be defined in a similar way as above. It is well known that problem (1.1)-(1.2) has a unique, nonnegative and bounded weak solution, at least locally in time $[16,17]$. Now we state the comparison principle for problem (1.1)-(1.2).

Lemma $2.1[16,17]$ Suppose that for $0<\tau<T, \bar{u}(x, t), \underline{u}(x, t) \in C\left(\mathbb{R}^{N} \times[0, T)\right) \cap L^{\infty}\left(\mathbb{R}^{N} \times\right.$ $[0, \tau])$ are supersolution and subsolution of the problem (1.1)-(1.2), respectively. If

$$
\bar{u}(x, 0) \geq \underline{u}(x, 0) \quad \text { for } x \in \mathbb{R}^{N},
$$

then, for all $(x, t) \in \mathbb{R}^{N} \times(0, T)$,

$$
\bar{u}(x, t) \geq \underline{u}(x, t) .
$$

To study the asymptotic behavior of solutions for problem (1.1)-(1.2), we adopt the space $X_{0}$ and $L^{\infty}\left(\rho_{\sigma}\right)$ as that in [16-18]. For any $\sigma>0$ and $\rho_{\sigma}(x)=\left(1+|x|^{2}\right)^{\frac{\sigma}{2}}$, the $L^{\infty}\left(\rho_{\sigma}\right)$ is defined as

$$
L^{\infty}\left(\rho_{\sigma}\right) \equiv\left\{\varphi ; \varphi \rho_{\sigma} \in L^{\infty}\left(\mathbb{R}^{N}\right)\right\}
$$

with the obvious norm $\|\varphi\|_{L^{\infty}\left(\rho_{\sigma}\right)}=\left\|\varphi \rho_{\sigma}\right\|_{L^{\infty}\left(\mathbb{R}^{N}\right)}$ and the $X_{0}$ is given by

$$
X_{0} \equiv\left\{\varphi \in L_{\text {loc }}^{1}\left(\mathbb{R}^{N}\right) ;\|\varphi\|_{1}<\infty \text { and } \ell(\varphi)=0\right\}
$$

with the norm $\|\cdot\|_{1}$. Here

$$
\|\varphi\|_{r}=\sup _{R \geq r} R^{-\frac{N(m-1)+2}{m-1}} \int_{\{|x| \leq R\}}|\varphi(x)| \mathrm{d} x \quad \text { and } \quad \ell(\varphi)=\lim _{r \rightarrow \infty}\|\varphi\|_{r} .
$$


Hence they are both Banach spaces. The existence and uniqueness of a weak solution of problem (1.4)-(1.5) with the initial-value $u_{0} \in X_{0}$ is shown in [16, 17], and this solution satisfies the following proposition.

Proposition 2.1 [17] Problem (1.4)-(1.5) generates a continuous bounded semigroup in $X_{0}$ given by

$$
S(t): w_{0} \rightarrow w(x, t)
$$

In other words, $S(t) w_{0} \in C\left([0, \infty) ; X_{0}\right)$. Moreover, if $u_{0} \in L^{1}\left(\mathbb{R}^{N}\right)$, then the semigroup $S(t)$ is a contraction.

We now introduce the definitions of scalings and the commutative relations between the semigroup operators and the dilation operators. For any $\mu, \beta>0$ and $\nu(x) \in X_{0}$, the dilation $D_{\lambda}^{\mu, \beta}$ is defined as follows:

$$
D_{\lambda}^{\mu, \beta} w(x) \equiv \lambda^{\mu} w\left(\lambda^{\beta} x\right)
$$

From the definitions of the dilation operator and the semigroup operator, we can get that for $\mu, \beta>0$ and $w_{0} \in X_{0}$,

$$
D_{\lambda}^{\mu, \beta}\left[S\left(\lambda^{2} t\right) w_{0}\right](x)=S\left(\lambda^{2-\mu(m-1)-2 \beta} t\right)\left[D_{\lambda}^{\mu, \beta} w_{0}\right](x)
$$

see details in $[19,20]$.

In the rest of this section, we give a sufficient condition for the existence of global solutions of problem (1.1)-(1.2) and establish the upper bounded estimates of these solutions.

Theorem 2.1 Let $\frac{2}{p-m}<\sigma<N$ and $M>0$. There exists a constant $\eta(M)$ such that for any $0 \leq \eta \leq \eta(M), \phi(x) \geq 0$ and $\|\phi\|_{L^{\infty}\left(\rho_{\sigma}\right)} \leq M$, the solutions $u(x, t)$ of problem (1.1)-(1.2) with the initial value $u_{0}(x)=\eta \phi(x)$ are global. Moreover, the following estimate holds:

$$
0 \leq u(x, t) \leq C(M, \eta)\left(1+t^{\frac{2}{\sigma(m-1)+2}}+|x|^{2}\right)^{-\frac{\sigma}{2}},
$$

where $C(M, \eta)$ is a constant dependent only on $M$ and $\eta$.

Remark 2.1 Notice that if $0 \leq \varphi \in C_{b}\left(\mathbb{R}^{N}\right)$ and $\limsup _{|x| \rightarrow \infty} \varphi(x)|x|^{\sigma}<\infty$, then $\varphi \in$ $L^{\infty}\left(\rho_{\sigma}\right)$. So, our results capture Theorem 3 in [6]. Here we use some ideas of them.

Proof To prove this theorem, we need the fact that if $v_{0}=M|x|^{-\sigma}$, then

$$
S(t) v_{0}(x) \leq C(M)\left(t^{\frac{2}{\sigma(m-1)+2}}+|x|^{2}\right)^{-\frac{\sigma}{2}}
$$

which has been given in Lemma 2.6 of [20]. We give the proof here for completeness. In fact,

$$
\left\|v_{0}\right\|_{r}=\sup _{R \geq r} R^{-\frac{N(m-1)+2}{m-1}} \int_{B_{R}} A|x|^{-\sigma} \mathrm{d} x \leq C r^{-\sigma-\frac{2}{m-1}} \rightarrow 0 \quad \text { as } r \rightarrow \infty .
$$


This means that $v_{0} \in X_{0}$. Therefore, from Proposition 2.1, we obtain that $S(t) v_{0}(x)$ is well defined. Taking $\mu=\frac{2 \sigma}{\sigma(m-1)+2}$ and $\beta=\frac{2}{\sigma(m-1)+2}$ in (2.2), we have

$$
\lambda^{\frac{2 \sigma}{\sigma(m-1)+2}}\left[S\left(\lambda^{2} s\right) v_{0}\right]\left(\lambda^{\frac{2}{\sigma(m-1)+2}} x\right)=S(s)\left[\lambda^{\frac{2 \sigma}{\sigma(m-1)+2}} v_{0}\left(\lambda^{\frac{2}{\sigma(m-1)+2}} \cdot\right)\right](x)=S(s) v_{0}(x) .
$$

Now taking $s=1, \lambda=t^{\frac{1}{2}}$ and $g(x)=S(1) v_{0}(x)$ in (2.5), we obtain that

$$
S(t) v_{0}(x)=t^{-\frac{\sigma}{\sigma(m-1)+2}} g\left(t^{-\frac{1}{\sigma(m-1)+2}} x\right) .
$$

The fact that $\phi \in C^{\infty}\left(\mathbb{R}^{N} \backslash\{0\}\right)$ clearly means that

$$
S(t) v_{0} \in C\left([0, \infty) \times \mathbb{R}^{N} \backslash\{(0,0)\}\right) \cap C^{\frac{\alpha}{2}, \alpha}\left((0, \infty) \times \mathbb{R}^{N}\right) \quad \text { for some } \alpha>0 ;
$$

see [21]. This implies that for $|x|=1$, the following limit holds:

$$
t^{-\frac{\sigma}{\sigma(m-1)+2}} g\left(t^{-\frac{1}{\sigma(m-1)+2}} x\right)=S(t) v_{0}(x) \rightarrow \phi(x)=M|x|^{-\sigma}=M \quad \text { as } t \rightarrow 0 .
$$

Let

$$
y=t^{-\frac{1}{\sigma(m-1)+2}} x
$$

So,

$$
|y| \rightarrow \infty \quad \text { as } t \rightarrow 0
$$

Therefore,

$$
|y|^{\sigma} g(y)-M \rightarrow 0
$$

as $|y| \rightarrow \infty$. This means that there exists an $M_{1}>1$ such that if $|y| \geq M_{1}$, then

$$
g(y) \leq 2 M|y|^{-\sigma}
$$

From (2.7), for $|y| \leq M_{1}$, there exists a constant $C$ such that

$$
g(y) \leq C
$$

Combining (2.8) and (2.9), we have

$$
g(x) \leq C(M)\left(1+|x|^{2}\right)^{-\frac{\sigma}{2}} \quad \text { for } x \in \mathbb{R}^{N}
$$

By (2.6), we thus obtain that

$$
S(t) v_{0}(x) \leq C(M)\left(t^{\frac{2}{\sigma(m-1)+2}}+|x|^{2}\right)^{-\frac{\sigma}{2}} .
$$


So, we complete the proof of (2.4). Now taking

$$
\varphi(x)=M\left(1+|x|^{2}\right)^{-\frac{\sigma}{2}},
$$

we get that

$$
0<\varphi(x) \leq v_{0}(x)=M|x|^{-\sigma} \quad \text { for } x \neq 0 .
$$

Therefore, by the comparison principle and (2.4), for all $t \geq 0$, we have

$$
S(t) \varphi(x) \leq S(t) v_{0}(x) \leq C(M)\left(t^{\frac{2}{\sigma(m-1)+2}}+|x|^{2}\right)^{-\frac{\sigma}{2}} .
$$

Since $S(t) \varphi(x) \in C\left([0, \infty) \times \mathbb{R}^{N}\right)$ (see [17, 21]), there exists a $t_{1}>0$ such that for all $|x| \leq 1$ and $0 \leq t \leq t_{1}$,

$$
S(t) \varphi(x) \leq C \varphi(x) \leq C(M)\left(1+|x|^{2}\right)^{-\frac{\sigma}{2}} .
$$

Combining this with (2.6) and using the comparison principle, we can get

$$
S(t) \phi(x) \leq S(t) \varphi(x) \leq C(M)\left(1+t^{\frac{2}{\sigma(m-1)+2}}+|x|^{2}\right)^{-\frac{\sigma}{2}} .
$$

In other words,

$$
S(t) \phi(x) \leq C(M)\left((1+t)^{\frac{2}{\sigma(m-1)+2}}+|x|^{2}\right)^{-\frac{\sigma}{2}} .
$$

If $\eta=0$, (2.3) clearly holds. In the rest of proof, we can assume that $\eta>0$. The hypothesis $\frac{2}{p-m}<\sigma<N$ indicates

$$
\sigma(p-m)-2>0 .
$$

Let

$$
\begin{aligned}
\eta(M)^{m-p} & =2 C^{p-1}(M)(p-m) \int_{0}^{\infty}(1+t)^{-\frac{\sigma(p-1)}{\sigma(m-1)+2}} \mathrm{~d} t \\
& =\frac{2 C(M)^{p-1}(\sigma(m-1)+2)(p-m)}{\sigma(p-m)-2}>0,
\end{aligned}
$$

where $C(M)$ is the constant given by (2.11). For $0<\eta \leq \eta(M)$, taking

$$
\alpha(t)=\left[\left(\eta^{m-p}-C(M)^{p-1}(p-m) \int_{0}^{t}(1+s)^{-\frac{\sigma(p-1)}{\sigma(m-1)+2}} \mathrm{~d} x\right)\right]^{-\frac{1}{p-m}},
$$

and

$$
w(x, t)=S(t) \phi(x),
$$


we obtain from (2.11) that $\alpha(t)$ is an increasing function satisfying

$$
\left\{\begin{array}{l}
a(0)=\eta \\
a(t) \leq 2^{\frac{1}{p-m}} \eta(M) \quad \text { for all } t \geq 0 \\
a^{\prime}(t)=C(M)^{p-1} a(t)^{p-m+1}(1+t)^{-\frac{\sigma(p-1)}{\sigma(m-1)+2}} \geq a(t)^{p-m+1}\|w(t)\|_{L^{\infty}\left(\mathbb{R}^{N}\right)}^{p-1}
\end{array}\right.
$$

Now letting $b(t)$ to satisfy

$$
\left\{\begin{array}{l}
b^{\prime}(t)=a(b(t))^{m-1} \\
b(0)=0
\end{array}\right.
$$

and then taking

$$
\bar{w}(x, t)=a(b(t)) w(x, t)
$$

one can see that $\bar{w}(x, t)$ is a supersolution of the following problem:

$$
\begin{aligned}
& \frac{\partial u}{\partial t}-\Delta u^{m}=u^{p}, \quad(x, t) \in \mathbb{R}^{N} \times(0, \infty) ; \\
& u(x, 0)=u_{0}=\eta \phi(x), \quad x \in \mathbb{R}^{N} .
\end{aligned}
$$

Therefore,

$$
\begin{aligned}
u(x, t) & \leq a(b(t)) w(x, t) \leq 2^{\frac{1}{p-m}} \eta(M) w(x, b(t)) \\
& \leq C(\eta, M)\left(1+b(t)^{\frac{2}{\sigma(m-1)+2}}+|x|^{2}\right)^{-\frac{\sigma}{2}} .
\end{aligned}
$$

(2.12) and (2.13) clearly mean that

$$
\eta^{m-1} t \leq b(t) \leq 2^{\frac{m-1}{p-m}} \eta(M)^{m-1} t .
$$

From this and (2.14), we can get (2.3). So, we complete the proof of this theorem.

\section{Complicated asymptotic behavior}

For any $M>0$, let $\eta(M)$ be as given by Theorem 2.1. We introduce

$$
B_{\eta(M)}^{\sigma,+} \equiv\left\{\varphi(x)=\eta \phi(x): \phi(x) \geq 0,\|\phi\|_{L^{\infty}}\left(\rho_{\sigma}\right) \leq M \text { and } 0 \leq \eta \leq \eta(M)\right\}
$$

and

$$
C_{\eta(M)}^{\sigma,+} \equiv\left\{\varphi \in C\left(\mathbb{R}^{N}\right) ; \varphi \in B_{\eta(M)}^{\sigma,+}\right\}
$$

In the rest of this section, we show that the complexity may occur in the asymptotic behavior of solutions of problem (1.1)-(1.2) with $u_{0} \in C_{\eta(M)}^{\sigma,+}$. Our main result is the following theorem. 
Theorem 3.1 Let $p>m+\frac{2}{N}$ and $\frac{2}{p-m}<\sigma<N$. Then there is a function $u_{0} \in C_{\eta(M)}^{\sigma,+}$ such that for any $\varphi \in C_{\eta(M)}^{\sigma,+}$, there exists a sequence $t_{n} \rightarrow \infty$ as $n \rightarrow \infty$ such that

$$
\lim _{n \rightarrow \infty} t_{n}^{\frac{\sigma}{\sigma(m-1)+2}} u\left(t_{n}^{\frac{1}{\sigma(m-1)+2}} x, t_{n}\right)=S(1) \varphi(x)
$$

uniformly on $\mathbb{R}^{N}$. Here $u(x, t)$ is the solution of problem (1.1)-(1.2).

To get this theorem, we need to prove the following lemma first.

Lemma 3.1 Suppose $p>m+\frac{2}{N}$ and $M>0$. Let $u$ be a solution of problem (1.1)-(1.2). If $0 \leq u_{0} \in B_{\eta(M)}^{\sigma,+}$ with $\frac{2}{p-m}<\sigma<N$, then

$$
\lim _{t \rightarrow \infty} t^{\frac{\sigma}{2+\sigma(m-1)}}\left\|u\left(t^{\frac{1}{2+\sigma(m-1)}} \cdot, t\right)-\left[S(t) u_{0}\right]\left(t^{\frac{1}{2+\sigma(m-1)}} \cdot\right)\right\|_{L^{\infty}\left(\mathbb{R}^{N}\right)}=0 .
$$

Proof We first define the functions

$$
u_{\lambda}(x, t)=D_{\lambda}^{\mu, \beta} u(x, \lambda t)=\lambda^{\mu} u\left(\lambda^{\beta} x, \lambda^{2} t\right)
$$

and

$$
w_{\lambda}(x, t)=D_{\lambda}^{\mu, \beta} w(x, \lambda t)=\lambda^{\mu} w\left(\lambda^{\beta} x, \lambda^{2} t\right),
$$

where $\mu=\frac{2 \sigma}{\sigma(m-1)+2}$ and $\beta=\frac{2}{\sigma(m-1)+2}$. Using the comparison principle, we know that for $(x, t) \in \mathbb{R}^{N} \times(0, \infty)$,

$$
w(x, t) \leq u(x, t)
$$

and for all $\lambda \geq 1$,

$$
w_{\lambda}(x, t) \leq u_{\lambda}(x, t)
$$

The results of Theorem 2.1 imply that

$$
\begin{aligned}
u_{\lambda}(x, t) & \leq C \lambda^{\mu}\left[\left(1+\lambda^{2} t\right)^{\beta}+\lambda^{2 \beta}|x|^{2}\right]^{-\frac{\sigma}{2}} \\
& \leq C\left(\left(\lambda^{-2}+t\right)^{2 \beta}+|x|^{2}\right)^{-\frac{\sigma}{2}} \\
& \leq C\left(\lambda^{-2}+t\right)^{-\mu}\left(1+\left(\lambda^{-2}+t\right)^{-2 \beta}|x|^{2}\right)^{-\frac{\sigma}{2}} .
\end{aligned}
$$

Here we have used the fact $\mu=\beta \sigma$. So,

$$
\int_{0}^{\tau} \int_{B_{1}} u_{\lambda}(x, t) \mathrm{d} x \mathrm{~d} t \leq C \int_{\lambda^{-2}}^{\tau+\lambda} s^{N \beta-\mu} \mathrm{d} s \int_{0}^{s^{-\beta}} r^{N-\sigma-1} \mathrm{~d} r \leq C \tau
$$

Now we estimate the integral

$$
\int_{0}^{\tau} \int_{B_{1}} u_{\lambda}(x, t)^{q} \mathrm{~d} x \mathrm{~d} t
$$


with $q>1$ in several steps. For any $\tau>0$, we take $\lambda$ large enough to satisfy $\lambda^{-2} \leq \tau$ and assume, without loss of generality, that $\left(\tau+\lambda^{-2}\right)^{-\beta}>1$ in the rest of this proof. Then using the same method as above, we have

$$
\int_{0}^{\tau} \int_{B_{1}} u_{\lambda}(x, t)^{q} \mathrm{~d} x \mathrm{~d} t \leq \begin{cases}C \tau^{2 \gamma \beta}+C \tau & \text { if } \gamma>0 \text { and } N \neq \sigma q, \\ C \tau+C \tau \ln \frac{1}{\tau} & \text { if } \gamma>0 \text { and } N=\sigma q, \\ C \tau+C \ln \left(1+\lambda^{2} \tau\right) & \text { if } \gamma=0, \\ C \tau+C \lambda^{-2 \beta \gamma} & \text { if } \gamma<0,\end{cases}
$$

where $\gamma=N+\sigma(m-1)-\sigma q+2$. Similarly, we can get the integral estimates for $w_{\lambda}(x, t)$, which have been given in [22]. By using the same methods as in [15], we can get that for $T>0$

$$
u_{\lambda}(T)-w_{\lambda}(T) \rightarrow 0 \quad \text { as } \lambda \rightarrow \infty
$$

uniformly on any compact subset of $\mathbb{R}^{N}$. For any $T, \lambda, \epsilon>0$, we can obtain from (3.1) that there exists a constant $R>0$ satisfying

$$
\left\|u_{\lambda}(T)\right\|_{L^{\infty}\left(\mathbb{R}^{N} \backslash B_{R}\right)} \leq\left\|\varphi_{\lambda}(T)\right\|_{L^{\infty}\left(\mathbb{R}^{N} \backslash B_{R}\right)}<\frac{\epsilon}{3}
$$

and

$$
\left\|w_{\lambda}(T)\right\|_{L^{\infty}\left(\mathbb{R}^{N} \backslash B_{R}\right)} \leq\left\|u_{\lambda}(\cdot, T)\right\|_{L^{\infty}\left(\mathbb{R}^{N} \backslash B_{R}\right)}<\frac{\epsilon}{3},
$$

where $\varphi_{\lambda}(x, t)=C \lambda^{\mu}\left[\left(1+\lambda^{2} t\right)^{\frac{2}{\sigma(m-1)+1}}+\left|\lambda^{\beta} x\right|^{2}\right]^{-\frac{\sigma}{2}}=C\left[\left(\lambda^{-2}+t\right)^{\frac{2}{\sigma(m-1)+2}}+|x|^{2}\right]^{-\frac{\sigma}{2}}$ and $B_{R} \equiv$ $\left\{x \in \mathbb{R}^{N} ;|x| \leq R\right\}$. Taking $R$ as given by (3.4), from (3.3), there exists $\lambda_{1}$ such that for all $\lambda \geq \lambda_{1}$

$$
\left\|u_{\lambda}(T)-w_{\lambda}(T)\right\|_{L^{\infty}\left(B_{R}\right)}<\frac{\epsilon}{3} .
$$

Therefore, from (3.4)-(3.6), we have

$$
\lim _{\lambda \rightarrow \infty}\left\|u_{\lambda}(T)-w_{\lambda}(T)\right\|_{L^{\infty}\left(\mathbb{R}^{N)}\right.}=0
$$

Now letting $T=1$ and $\lambda=t^{\frac{1}{2}}$ in (3.7), we get that

$$
\lim _{t \rightarrow \infty} \frac{\sigma}{\frac{\sigma}{2+\sigma(m-1)}}\left\|\left[u\left(t^{\frac{1}{2+\sigma(m-1)}} \cdot, t\right)-w\left(t^{\frac{1}{2+\sigma(m-1)}} \cdot t\right)\right]\right\|_{L^{\infty}\left(\mathbb{R}^{N)}\right.}=0 .
$$

So, we complete the proof of this lemma.

Now we can prove our main result.

Proof of Theorem 3.1 Let

$$
\mu=\frac{2 \sigma}{\sigma(m-1)+2}
$$


and

$$
\beta=\frac{2}{\sigma(m-1)+2}
$$

From the definition of $C_{\eta(M)}^{\sigma,+}$, we obtain that there exists a countable set $F$ such that

$$
F \subset C_{\eta(M)}^{\sigma,+} \cap L^{1}\left(\mathbb{R}^{N}\right)
$$

and for any $\epsilon>0$ and $\varphi \in C_{\eta(M)}^{\sigma,+}$, there exists a function $\phi_{\epsilon} \in F$ satisfying

$$
\left\|\varphi_{\epsilon}-\varphi\right\|_{L^{\infty}\left(\mathbb{R}^{N}\right)}<\epsilon
$$

Therefore, there exists a sequence $\left\{\varphi_{j}\right\}_{j \geq 1} \subset F$ such that

I. For any $\phi \in F$, there exists a subsequence $\left\{\varphi_{j_{k}}\right\}_{k \geq 1}$ of the sequence $\left\{\varphi_{j}\right\}_{j \geq 1}$ satisfying

$$
\varphi_{j_{k}}(x)=\phi \quad \text { for all } k \geq 1
$$

II. There exists a constant $C>0$ satisfying

$$
\max \left(\left\|\varphi_{j}\right\|_{L^{\infty}\left(\mathbb{R}^{N}\right)},\left\|\varphi_{j}\right\|_{L^{1}\left(\mathbb{R}^{N}\right)}\right) \leq C j \quad \text { for } j \geq 1
$$

Now we can follow the methods given in [9] to construct an initial value as follows. Let

$$
u_{0}(x)=\sum_{j=1}^{\infty} \lambda_{j}^{-\mu} \chi_{j}\left(x / \lambda_{j}^{\beta}\right) \varphi_{j}\left(x / \lambda_{j}^{\beta}\right)=\sum_{j=1}^{\infty} D_{\lambda_{j}^{-1}}^{\mu, \beta}\left[\chi_{j}(x) \varphi_{j}(x)\right]
$$

Here

$$
\lambda_{j}= \begin{cases}2 & \text { for } j=1, \\ \max \left(j^{\frac{4 N(m-1)+8}{2 N-\mu[N(m-1)+2]}} \lambda_{j-1}^{\frac{4 \beta N-2 \mu}{2 N-N(m-1)+2]}},\left(2^{j} \lambda_{j-1}\right)^{\frac{1}{\mu}}, \bar{\lambda}_{j}\right) & \text { for } j>1,\end{cases}
$$

$\chi_{j}(x)$ is the cut-off function defined on $\left\{x \in \mathbb{R}^{N} ; 2^{-j}<|x|<2^{j}\right\}$ relatively to $\left\{x \in \mathbb{R}^{N} ; 2^{-j+1}<\right.$ $\left.|x|<2^{j-1}\right\}$, and $\bar{\lambda}_{j}$ is selected large enough to satisfy

$$
\begin{aligned}
D_{\lambda_{j}}^{\mu, \beta}\left[S\left(\lambda_{j}^{2} t\right) u_{0}(x)\right]= & D_{\lambda_{j}}^{\mu, \beta}\left[S\left(\lambda_{j}^{2} t\right) \sum_{n=1}^{j-1} \lambda_{n}{ }^{-\mu} \chi_{n}\left(x / \lambda_{n}^{\beta}\right) \varphi_{n}\left(x / \lambda_{n}^{\beta}\right)\right] \\
& +D_{\lambda_{j}}^{\mu, \beta}\left[S\left(\lambda_{j}^{2} t\right) \lambda_{j}{ }^{-\mu} \chi_{j}\left(x / \lambda_{j}^{\beta}\right) \varphi_{j}\left(x / \lambda_{j}^{\beta}\right)\right] \\
& +D_{\lambda_{j}}^{\mu, \beta}\left[S\left(\lambda_{j}^{2} t\right) \sum_{n=j+1}^{\infty} \lambda_{n}{ }^{-\mu} \chi_{n}\left(x / \lambda_{n}^{\beta}\right) \varphi_{n}\left(x / \lambda_{n}^{\beta}\right)\right] .
\end{aligned}
$$

Notice first that if $\varphi \in C_{\eta(M)}^{\sigma,+}$, then

$$
\|\varphi\|_{L^{\infty}\left(\mathbb{R}^{N}\right)} \leq \eta(M), \quad\|\varphi\|_{L^{\infty}\left(\rho_{\sigma}\right)} \leq \eta(M)
$$


and

$$
\varphi \in C_{0}\left(\mathbb{R}^{N}\right)
$$

By (3.9) and (3.10), we have

$$
\left\|u_{0}\right\|_{L^{\infty}\left(\mathbb{R}^{N}\right)} \leq\left\|u_{0}\right\|_{L^{\infty}\left(\rho_{\sigma}\right)} \leq \sup _{j \geq 1}\left\|\lambda_{j}^{-\mu} \chi_{j}\left(x / \lambda_{j}^{\beta}\right) \varphi_{j}\left(x / \lambda_{j}^{\beta}\right)\right\|_{L^{\infty}\left(\rho_{\sigma}\right)} \leq \eta(M) .
$$

So, we have

$$
u_{0} \in C_{\eta(M)}^{\sigma,+} \subset C_{0}\left(\mathbb{R}^{N}\right)
$$

Using the same method as that in [9], we can get that for any $\varphi \in F$, there exists a sequence $t_{n} \rightarrow \infty$ as $n \rightarrow \infty$ such that

$$
t_{n}^{\frac{\sigma}{\sigma(m-1)+2}}\left[S\left(t_{n}\right) u_{0}\right]\left(t_{n}^{\frac{1}{\sigma(m-1)+2}} x\right) \stackrel{n \rightarrow \infty}{\longrightarrow} S(1) \varphi(x)
$$

uniformly on $\mathbb{R}^{N}$. For any $\phi \in C_{\eta(M)}^{\sigma,+}$, from (1.2), we know that there exists a sequence $\left\{\varphi_{k}\right\} \subset F$ such that

$$
\varphi_{k} \rightarrow \phi \quad \text { as } k \rightarrow \infty
$$

Therefore,

$$
S(1) \varphi_{k} \rightarrow S(1) \phi \quad \text { as } k \rightarrow \infty
$$

uniformly on any compact subset of $\mathbb{R}^{N}$. This uses the fact that the map $S(1)$ is regularizing since the images of bounded sets are relatively compact subsets of $C^{\alpha}$ for some $\alpha>0$ in compact sets of $\mathbb{R}^{N}$ [21]. And notice that $\varphi_{k}, \phi \in C_{\eta(M)}^{\sigma,+} \subset B_{\eta(M)}^{\sigma,+}$. We thus obtain from Theorem 2.1 that for any $\varepsilon>0$, there exists $R>0$ such that if $|x|>R$, then

$$
S(1) \phi(x)<\frac{\varepsilon}{3}
$$

and

$$
S(1) \varphi_{k}(x)<\frac{\varepsilon}{3} \quad \text { for all } k \geq 1
$$

Combining (3.12), (3.13) with (3.14), we thus have that

$$
S(1) \varphi_{k} \rightarrow S(1) \phi \quad \text { as } k \rightarrow \infty
$$

uniformly on $\mathbb{R}^{N}$. By Lemma 3.1, (3.11) and (3.15), we can get that for any $\phi \in C_{\eta(M)}^{\sigma,+}$, there exists a sequence $t_{n} \rightarrow \infty$ as $t \rightarrow \infty$ such that

$$
\lim _{n \rightarrow \infty} t_{n}^{\frac{\sigma}{\sigma(m-1)+2}} u\left(t_{n}^{\frac{1}{\sigma(m-1)+2}} x, t_{n}\right)=S(1) \phi(x)
$$

uniformly on $\mathbb{R}^{N}$. So, we complete the proof of Theorem 3.1. 


\section{Competing interests}

The authors declare that they have no competing interests.

\section{Authors' contributions}

The paper is the result of joint work of both authors who contributed equally to the final version of the paper. Both authors read and approved the final manuscript.

\section{Author details}

${ }^{1}$ School of Mathematics and Statistics, Chongqing Three Gorges University, Wanzhou, China. ${ }^{2}$ School of Mathematical Sciences, South China Normal University, Guangzhou, China.

\section{Acknowledgements}

This work is supported by NSFC, the Research Fund for the Doctoral Program of Higher Education of China, the Natural Science Foundation Project of 'CQ CSTC' (cstc2012jjA00013), the Scientific and Technological Projects of Chongqing Municipal Commission of Education (KJ121105).

\section{Received: 12 July 2012 Accepted: 4 February 2013 Published: 21 February 2013}

\section{References}

1. Galaktionov, VA, Kurdjumov, SP, Mihăiov, AP, Samarskiï, AA: On unbounded solutions of the Cauchy problem for the parabolic equation $u_{t}=\nabla\left(u^{\sigma} \nabla u\right)+u^{\beta}$. Sov. Math. Dokl. 252(6), 1362-1364 (1980) (Russian)

2. Galaktionov, VA: Blow-up for quasilinear heat equations with critical Fujita's exponents. Proc. R. Soc. Edinb. A 124(3), 517-525 (1994) (English summary)

3. Kawanago, T: Existence and behaviour of solutions for $u_{t}=\Delta\left(u^{m}\right)+u^{\ell}$. Adv. Math. Sci. Appl. 7(1), 367-400 (1997) (English summary)

4. Mochizuki, K, Suzuki, R: Critical exponent and critical blow-up for quasilinear parabolic equations. Isr. J. Math. 98 , 141-156 (1997) (English summary)

5. Suzuki, R: Asymptotic behavior of solutions of quasilinear parabolic equations with slowly decaying initial data. Adv Math. Sci. Appl. 9(1), 291-317 (1999) (English summary)

6. Mukai, K, Mochizuki, K, Huang, Q: Large time behavior and life span for a quasilinear parabolic equation with slowly decaying initial values. Nonlinear Anal. 39(1), 33-45 (2000)

7. Suzuki, R: Asymptotic behavior of solutions of quasilinear parabolic equations with supercritical nonlinearity. (English summary). J. Differ. Equ. 190(1), 150-181 (2003)

8. Vázquez, JL, Zuazua, E: Complexity of large time behaviour of evolution equations with bounded data. Chin. Ann. Math., Ser. B 23(2), 293-310 (2002)

9. Yin, J, Liangwei, W, Huang, R: Complexity of asymptotic behavior of solutions for the porous medium equation with absorption. Acta Math. Sci. 30(6), 1865-1880 (2010)

10. Cazenave, T, Dickstein, F, Weissler, FB: Universal solutions of the heat equation on $\mathbb{R}^{N}$. Discrete Contin. Dyn. Syst. 9(5), 1105-1132 (2003) (English summary)

11. Cazenave, T, Dickstein, F, Weissler, FB: Universal solutions of a nonlinear heat equation on $\mathbb{R}^{N}$. Ann. Sc. Norm. Super. Pisa, Cl. Sci. 2(1), 77-117 (2003) (English summary)

12. Cazenave, T, Dickstein, F, Weissler, FB: Chaotic behavior of solutions of the Navier-Stokes system in $\mathbb{R}^{N}$. Adv. Differ. Equ. 10(4), 361-398 (2005)

13. Cazenave, T, Dickstein, F, Weissler, FB: Nonparabolic asymptotic limits of solutions of the heat equation on $\mathbb{R}^{N}$. J. Dyn. Differ. Equ. 19(3), 789-818 (2007)

14. Carrillo, JA, Vázquez, JL: Asymptotic complexity in filtration equations. J. Evol. Equ. 7(3), 471-495 (2007) (English summary)

15. Kamin, S, Peletier, LA: Large time behaviour of solutions of the heat equation with absorption. Ann. Sc. Norm. Super Pisa, Cl. Sci. 12(3), 393-408 (1985)

16. Bénilan, P, Crandall, MG, Pierre, M: Solutions of the porous medium equation in $\mathbb{R}^{N}$ under optimal conditions on initial values. Indiana Univ. Math. J. 33(1), 51-87 (1984)

17. Vázquez, JL: The Porous Medium Equation. Mathematical Theory. Oxford Mathematical Monographs. Clarendon, Oxford (2007)

18. DiBenedetto, E, Herrero, MA: On the Cauchy problem and initial traces for a degenerate parabolic equation. Trans. Am. Math. Soc. 314(1), 187-224 (1989)

19. Yin, J, Wang, L, Huang, R: Complexity of asymptotic behavior of the porous medium equation in $\mathbb{R}^{N}$. J. Evol. Equ. 11(2), 429-455 (2011)

20. Wang, L, Yin, J, Jin, C: $\omega$-Limit sets for porous medium equation with initial data in some weighted spaces. Discrete Contin. Dyn. Syst., Ser. B 18(1), 223-236 (2013)

21. DiBenedetto, E: Degenerate Parabolic Equations. Springer, New York (1993)

22. Kamin, S, Peletier, LA: Large time behaviour of solutions of the porous media equation with absorption. Isr. J. Math. 55(2), 129-146 (1986)

doi:10.1186/1687-2770-2013-35

Cite this article as: Wang and Yin: Complicated asymptotic behavior of solutions for a porous medium equation with nonlinear sources. Boundary Value Problems 2013 2013:35. 